\title{
Common Material Foundation of Gravitation Theory and Quantum Theory
}

\author{
Zhou Shuwen \\ School of Physics, Huazhong University of Science and Technology, Wuhan, China
}

\section{Email address:}

zhousw123456@163.com

\section{To cite this article:}

Zhou Shuwen. Common Material Foundation of Gravitation Theory and Quantum Theory. American Journal of Modern Physics. Vol. 7, No. 3, 2018, pp. 103-115. doi: 10.11648/j.ajmp.20180703.11

Received: April 26, 2018; Accepted: June 4, 2018; Published: June 29, 2018

\begin{abstract}
In order to unite gravitational field with other fields, we must find the common material foundation of the gravitation theory and quantum theory. For the end, I propose the duality framework of the universe. The universe is composed of continuous universal continuum and separate p net; Universal energy Eu and various special energies Es that transform into one another at high frequency; Basic forces that dominate the universe are the universal compressive force and quantum dispersive force. The above duality framework, can explain naturally the wave-particle duality, probabilistic nature, uncertainty relation and quantum entanglement; no using Einstein's theory, can obtain the mass-energy relation, Einstein's equations, Friedmann's equation and the proportions of dark energy, dark matter and ordinary matter; includes, links and foreruns both the gravitation theory and quantum theory; is their common material foundation to give their unified harmonious image; and can solve the puzzlers about the singularity, non-locality, uncertainty of energy, et al . Daring predictions: The electromagnetic wave must have an adjoint strain wave. The gravitational wave must have an adjoint wave of matching field. It is in the wrong direction to find the particle of the "dark matter".
\end{abstract}

Keywords: Quantum Dispersive Force, Universal Compressive Force, Universal Lump Elasticity, $\mathrm{E}_{\mathrm{u}} \leftrightarrow \mathrm{E}_{\mathrm{s}}$ High Frequency Transformation, Complex Field

\section{Introduction}

Regarding the gravitational theory, quantum theory and universal theory, it can be said that currently a hundred schools of thought is contending for their advancement [1-8, 23-32]. What is the material foundation of the space? What is the essence of the wave-particle duality? What is the basic action in universe? All the problems are not clear. We meet with the puzzlers about the singularity, non-locality, uncertainty of energy, et al. For the end, our task is to find the common material foundation of the gravitation theory, quantum theory and universe theory to give their unified harmonious image.

\subsection{Hypothesis on Duality}

(1) The universe is made up of the continuous universal continuum and the separate $\mathrm{P}$ chamber network embedded therein $[5,6]$. The vacuum state $\mathrm{P}$ chamber radius is $r_{p}=\left(\hbar G / c^{3}\right)^{1 / 2}$, the quantum charge is $(\hbar c)^{1 / 2}[8,9]$ and mass is $\mathrm{m}_{\mathrm{p}}=(\hbar \mathrm{c} / \mathrm{G})^{1 / 2}$. The interior of the $P$ chamber is described with the multi-D superspace. The universal lump has elasticity [9, 44]. The elastic modulus is $\mathrm{E}_{\mathrm{e}}=\left(\mathrm{Gm}_{\mathrm{p}}{ }^{2} / \mathrm{r}_{\mathrm{p}}{ }^{2}\right) /\left(4 \pi \mathrm{r}_{\mathrm{p}}{ }^{2}\right)=\left(\mathrm{c}^{4} / \mathrm{G}\right) / 4 \pi \mathrm{r}_{\mathrm{p}}{ }^{2}$. The universe continuum is described with Riemann's geometry.

(2) There are two classes of motion and energy in the universe [7]. One is the $\mathrm{E}_{\mathrm{u}}$ energy possessed by each and every particle, $\mathrm{E}_{\mathrm{u}} \propto \mathrm{m}_{\mathrm{u}}$. $\mathrm{E}_{\mathrm{u}}$ is distributed in the universal continuum to form the $\mathrm{U}$ field. The other class are the $\mathrm{E}_{\mathrm{s}}$ energies possessed by various special forms of motion, $\mathrm{E}_{s}=\sum_{i} E_{s i}$ $E_{s i} \propto m_{s i}$, such as electromagnetic motion, flavor motion, color motion. The $\mathrm{E}_{\mathrm{s}}$ is only distributed in the $\mathrm{P}$ chamber. The $E_{u}$ and $E_{s}$ of each particle are transformed into each other at high frequency, while $\mathrm{E}_{\mathrm{T}}=\mathrm{E}_{\mathrm{u}}+\mathrm{E}_{\mathrm{s}}$ remains unchanged. The kinetic energy $\mathrm{E}_{\mathrm{k}}$ is $E_{u} \cap E_{s}$.

(3) Two basic actions [8]. Vacuum is the equilibrium state of $E_{u}$ and $E_{s}$ with the lowest energy. When there is excited $d_{m}$ superimposed in the universal continuum outside the $\mathrm{P}$ 
chamber, vacuum will give it universal compressive force $\mathrm{dF}_{\mathrm{u}}=\left(\mathrm{Gm}_{\mathrm{p}} / \mathrm{r}_{\mathrm{p}}{ }^{2}\right) \mathrm{dm}_{\mathrm{u}}$ that compresses it back into a certain $\mathrm{P}$ chamber and changes into $E_{s}$. When there is excited $d_{m}$ superimposed in the $\mathrm{p}$ chamber, vacuum will give it quantum dispersive surface force $\mathrm{dF}_{\mathrm{q}}=\left(\mathrm{Gm}_{\mathrm{p}} / \mathrm{r}_{\mathrm{p}}{ }^{2}\right) \mathrm{dm}_{\mathrm{s}}$ that compresses it back into the universal continuum and changes into $\mathrm{E}_{\mathrm{u}}$. When the $\mathrm{E}_{\mathrm{s}}$ excited in the $\mathrm{P}$ net is captivated in a sphere with radius $\mathrm{r}$, quantum dispersive surface force $\hbar c / \mathrm{r}^{2}$ will be generated [9].

\subsection{Duality Framework}

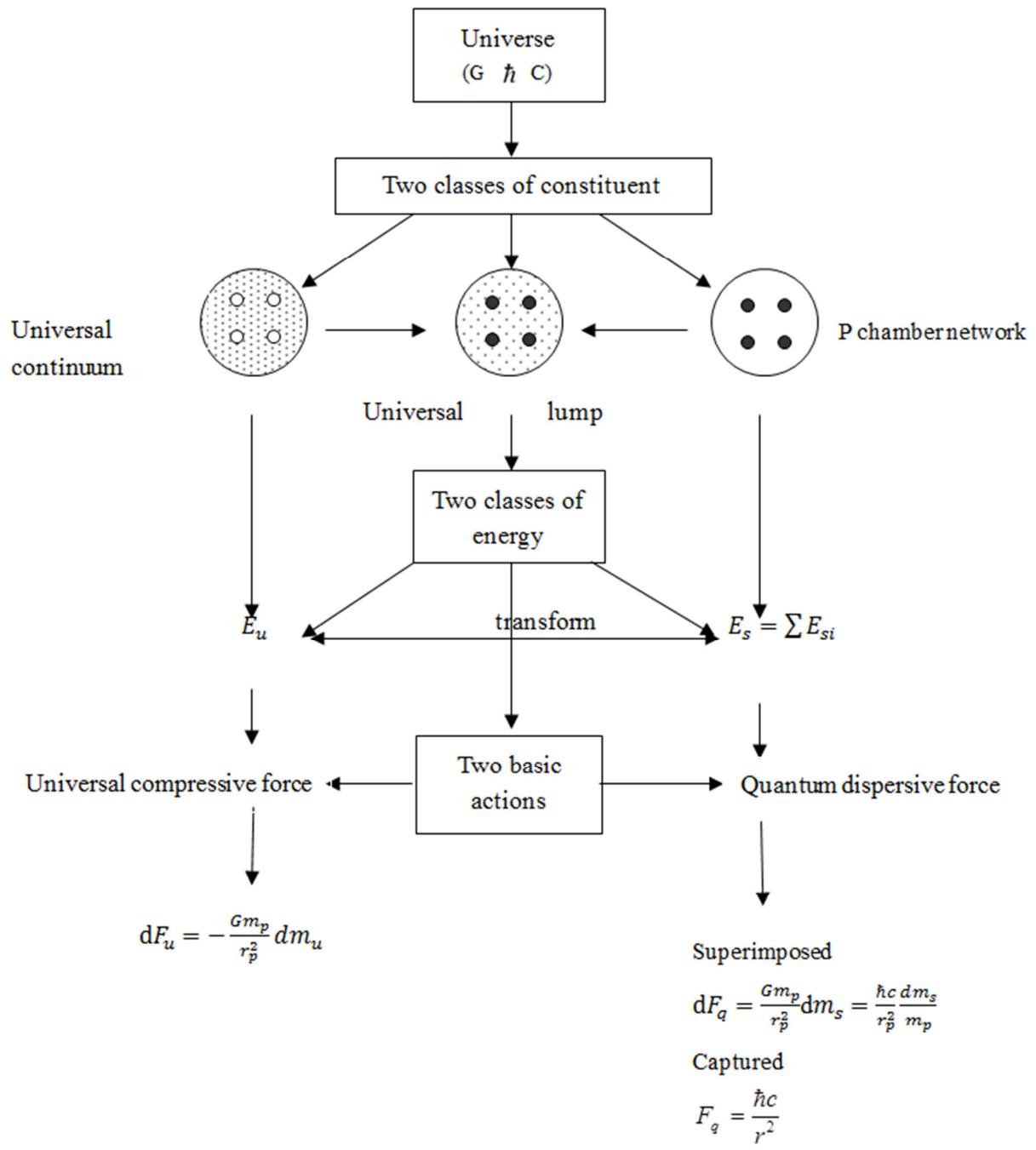

Figure 1. Duality framework.

\subsection{Generalized Coordinates}

For an isotropic homogeneous elastomer sphere, under the action of elastic surface force, when the variation of volum $\Delta \mathrm{V} \ll \mathrm{V}$, the equation of motion can be written as

$$
\begin{gathered}
\mathrm{m} \frac{d^{2}}{d t^{2}} q_{V}=-K q_{V} \\
q_{V} \equiv r_{e} \Sigma_{V} \\
\Sigma_{V} \equiv \frac{V-V_{0}}{V_{0}}
\end{gathered}
$$

where $m$ is the sphere's equivalent mass, $r_{e}$ and $V_{o}$ are the radius and volume in equilibrium state, and $\mathrm{k}$ is the elastic constant in volume deformation, $\Sigma_{V}$ the relative variation in volume, $q_{V}$ the generalized coordinate. $-\mathrm{k} q_{V}$ is the elastic surface force.

The generalized coordinates are defined as whatever $\mathrm{s}$ quantities that can define unique "position" of the system in "space". The morphology of Lagrangian equation has nothing to do with the choice of the coordinate. We popularize the generalized coordinate to the universal lump spheres acted on only by the surface forces $\mathrm{F}_{\mathrm{q}}$ and $\mathrm{F}_{\mathrm{u}}$.

Definitions

$$
\begin{gathered}
q \equiv r_{e} \Sigma \\
\Sigma \equiv \frac{\Delta u}{u_{0}}
\end{gathered}
$$

where $r_{e}$ is the characteristic radius of the system in equilibrium state, $\mathrm{u}$ the physical quantity describing the state 
of the system while $\Delta \mathrm{u}$ is the deviation of $\mathrm{u}$ from equilibrium state. $u_{\mathrm{o}}$ is the absolute value of equilibrium value, $\Sigma$ is the relative variation of $u$.

The system's Lagrangian function is

$$
L=\frac{1}{2} m \dot{q}^{2}-E_{P}(q)
$$

Further,

$$
\frac{\partial L}{\partial q}=-\frac{\partial E_{p}}{\partial q}=F\left(F_{q}, F_{u}\right)
$$

Lagrangian equation can be written as

$$
\mathrm{m} r_{e} \frac{d^{2}}{d t^{2}} \Sigma=F\left(F_{q}, F_{u}\right) .
$$

\section{2. $E_{\mathrm{u}}$ and $E_{\mathrm{s}}$ Oscillation of the $P$ Sphere}

\subsection{P Sphere Mechanical System}

The $\mathrm{P}$ chamber constitutes a mechanical system with its surrounding universal continuum, or $\mathrm{P}$ sphere for short. The vacuum state $P$ chamber's mass, energy and radius are $m_{p}, E_{P}$, $r_{p}$, respectively. The excited energy $E_{s}$ and $E_{u}$, are distributed in the chamber and in the universal continuum outside the chamber, respectively.

For simplicity, Let us assume that vacuum is the static equilibrium state, $E_{p}$ not enters into the energy transformation.

Definitions:

$$
\begin{gathered}
\Sigma_{p} \equiv \frac{E_{u}-E_{s}}{E_{p}} \\
q_{p} \equiv r_{e} \Sigma_{p}=r_{p}\left(\frac{E_{u}-E_{s}}{E_{p}}\right)
\end{gathered}
$$

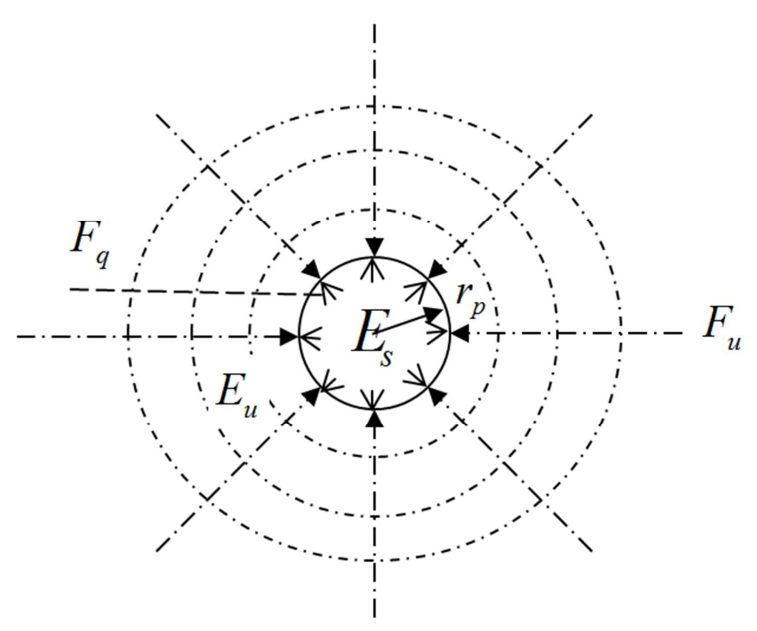

Figure 2. P sphere acted on by surface forces.

As show in figure 2, the interior and exterior of the $p$ chamber interface bear the surface forces of $F_{q}$ and $F_{u}$, respectively.

$$
F_{p}=-\frac{G m_{p}}{r_{p}^{2}} \cdot m_{u}+\frac{\hbar c}{r_{p}^{2}} \frac{m_{s}}{m_{p}}=-\frac{G m_{p}^{2}}{r_{p}^{2}} \frac{\left(m_{u}-m_{s}\right)}{m_{p}}
$$

$\mathrm{E} \propto \mathrm{m}$, so

$$
F_{P}=-\frac{G m_{p}^{2}}{r_{p}^{2}} \frac{\left(E_{u}-E_{s}\right)}{E_{p}}
$$

\subsection{Equations of Motion}

The $\mathrm{P}$ spheres equations of motion is

$$
\begin{gathered}
m_{p} r_{p} \frac{d^{2}}{d \tau^{2}}\left(\frac{E_{u}-E_{S}}{E_{p}}\right)=-\frac{G m_{p}^{2}}{r_{p}^{2}}\left(\frac{E_{u}-E_{S}}{E_{p}}\right) \\
\frac{d^{2}}{d \tau^{2}}\left(\frac{E_{u}-E_{S}}{E_{p}}\right)=-\omega_{p}^{2}\left(\frac{E_{u}-E_{S}}{E_{p}}\right), \omega_{p}^{2}=\frac{c^{5}}{\hbar G}
\end{gathered}
$$

The Lagrangian function is

$$
L_{P}=\frac{1}{2} m_{P} \dot{q}_{p}^{2}-\frac{1}{2} m_{p} \omega_{p}^{2} q_{p}^{2}
$$

The solutions are

$$
\begin{gathered}
E_{u}=\frac{E_{T}}{2}\left(1-\cos \omega_{P} \tau\right), \\
E_{s}=\frac{E_{T}}{2}\left(1+\cos \omega_{P} \tau\right), E_{s}=\sum_{i} E_{s i} \\
E_{T}=E_{u}+E_{s}=2 \overline{E_{u}}=2 \overline{E_{S}} .
\end{gathered}
$$

\section{3. $E_{u}$ and $E_{s}$ Oscillation of Particle}

(1) The energy becomes $E_{s}$ upon entering the P chamber via the interface and becomes $E_{u}$ when emitted out transforming at high frequency. The particle is localized $\left(E_{S}\right)$, also nonlocalized $\left(\mathrm{E}_{\mathrm{u}}\right)$. Each particle is possessing of the total energy $E_{T}=2 \overline{E_{u}}=2 \overline{E_{s}}$, what we have obtained by U means measurement or $\mathrm{S}$ means is but the mean value $\overline{E_{u}}$ or $\overline{E_{S}}$

(2) In $E_{u} \leftrightarrow E_{s}$ oscillation, the universe lump is deformed but without much move. Energy $E_{u}$ is transmitted with superluminal speeds, just like electric energy is transmitted in a conductor with a speed exceeding that of electrons move. The $\mathrm{P}$ network doesn't move. By the move of particle is meant the excited energy $E_{s}$ transforming via $E_{u}$ from a $P$ chamber pressed into another probabilistically that is the jump of $E_{s}$ energy. This is just like the "jump" of flashing jumpingly bulb with no move of the bulbs. The average speed of a long period of probability jump of $\mathrm{E}_{\mathrm{s}}$ in the $\mathrm{P}$ network is $\overline{\mathrm{u}} \leq \mathrm{c}$.

(3) Regarding the character of probability: After energy $E_{s}$ changes into $E_{u}$ and back to $E_{s}$ again, what $P$ chamber is it after all that it has returned to is probabilistic, with the probabilistic density proportional to the $E_{u}$ 's energy density $\rho_{u}$ at that. In the P chamber, within $E_{u} \rightarrow E_{s}$ on the premise of conservation of the relevant physical quantity, what is the $\mathrm{E}_{\mathrm{si}}$ that has been converted into is also probabilistic. The character of probability in quantum mechanics originates in the highly frequent transformation $E_{u} \leftrightarrow E_{s}$. 


\section{U Field in the Universal Continuum}

\subsection{Universal Lump Elasticity}

(1) The action of $\mathrm{P}$ chamber on universal continuum The $E_{u}$ and $E_{s}$ are transformed into each other at high frequency. The average of a long period is corresponding to the value of the static equilibrium.. According to above, the universal lump in vacuum state is an isotropic homogeneous elastomer, a single $\mathrm{P}$ chamber excited state excites spherically symmetrical gravitational field, or $\mathrm{U}$ field for short. As shown in figure 3 , in the spherical layer $\left(\mathrm{r}_{\mathrm{p}}-\mathrm{r}\right)$, its inner surface bears the quantum dispersive force in the $\mathrm{P}$ chamber, that is, $\mathrm{F}_{\mathrm{q}}=\left(\mathrm{Gm}_{\mathrm{p}} / \mathrm{r}_{\mathrm{p}}{ }^{2}\right)\left(\mathrm{m}_{\mathrm{p}}+\mathrm{m}_{\mathrm{s}}\right)$; while the outer surface bears the universal compressive force $F_{u}=\sigma_{r r}^{T} 4 \pi r^{2}$.

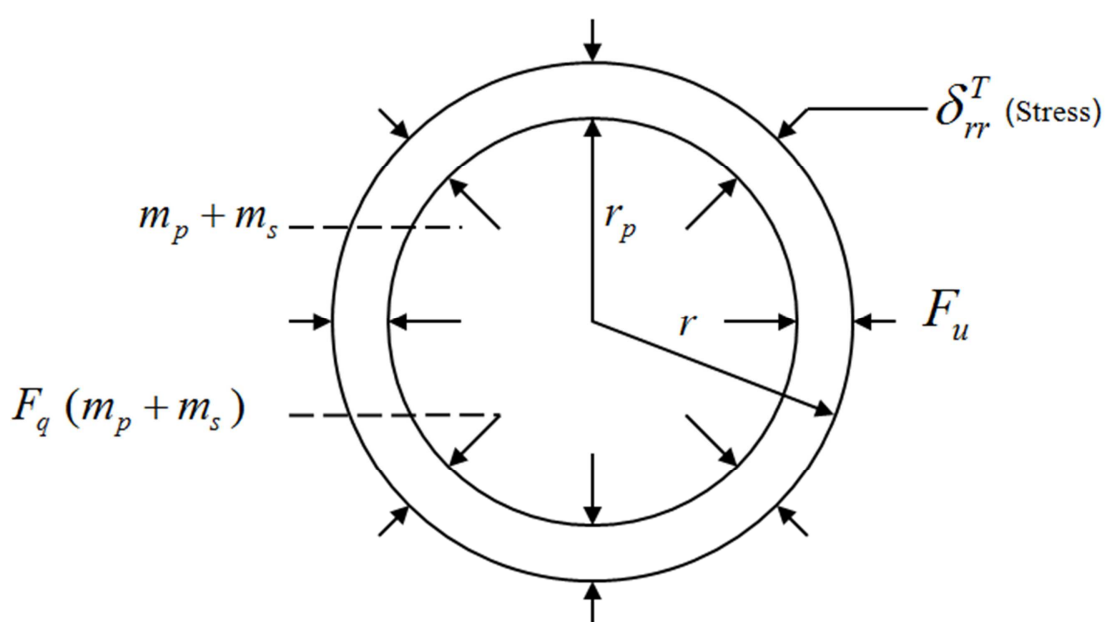

Figure 3. Universal lump spherical layer acted on by surface forces.

The static equilibrium equation is

$$
\frac{G m_{P}}{r_{P}^{2}}\left(m_{P}+m_{s}\right)+\sigma_{r r}^{T} 4 \pi r^{2}=0
$$

Further

$$
\sigma_{r r}^{T}=-\frac{G m_{p}^{2}}{4 \pi r_{p}^{2}}\left(1+\frac{m_{s}}{m_{p}}\right) r^{-2}
$$

The universal lump elastomer's Hooke equation is

$$
\varepsilon_{r r}^{T}=E_{e}^{-1} \sigma_{r r}^{T} .
$$

Strain and displacement are

$$
\begin{aligned}
& \varepsilon_{r r}^{T}=-\frac{r_{P}^{2}}{r^{2}}\left(1+\frac{m_{s}}{m_{P}}\right), u_{r}^{T}=r_{P} \frac{r_{P}}{r}\left(1+\frac{m_{s}}{m_{P}}\right) . \\
& E_{u}\left(m_{s}\right)=\int_{r_{P}}^{\infty} \frac{G m_{P} m_{s}}{4 \pi r^{4}} \cdot 4 \pi r^{2} d r=m_{s} C^{2}=m_{u} \sqrt{G} \cdot \frac{C^{2}}{\sqrt{G}}=m_{s} \sqrt{G} \frac{\sqrt{\hbar c}}{r_{P}}=\frac{m_{s}}{m_{P}} \sqrt{\hbar c} \cdot \frac{\sqrt{\hbar c}}{r_{P}}
\end{aligned}
$$

Where $\mathrm{m}_{\mathrm{s}}=\mathrm{m}_{\mathrm{u}}=\mathrm{m}$ [see Eq.(18)].

That is, $\mathrm{mc}^{2}$ is the potential energy interacted between mass charge $\mathrm{m}_{\mathrm{u}} \mathrm{G}^{1 / 2}$ and vacuum potential $\mathrm{c}^{2} / \mathrm{G}^{1 / 2}$, or that between quantum charge $\left(\mathrm{m}_{\mathrm{s}} / \mathrm{m}_{\mathrm{p}}(\mathrm{hc})^{1 / 2}\right.$ and vacuum potential $(\mathrm{hc})^{1 / 2} / \mathrm{r}_{\mathrm{p}}$. The relativity only has the result $\mathrm{mc}^{2}$, but not the explanation on the mechanism. The opinion on the transformation of the mass and energy is mistaken. The rest energy of the elementary particle is $\mathrm{E}_{\mathrm{T}}=\mathrm{E}_{\mathrm{u}}+\mathrm{E}_{\mathrm{s}}=2 \mathrm{mc}^{2}$ [see Eq.(18)].

Similarly, we have

$$
\sigma_{r r}^{T}\left(m_{s}\right)=-\frac{G m_{P} m_{s}}{4 \pi r_{P}^{2} r^{2}},
$$

The density of universal continuum elastic energy is

$$
\rho_{u}^{T}\left(m_{P}+m_{s}\right)=\frac{1}{2} E_{e}^{-1}\left(\sigma_{r r}^{T}\right)^{2}=\frac{G m_{P}^{2}}{8 \pi r^{4}}+\frac{G m_{P} m_{s}}{4 \pi r^{4}}+\frac{G m_{s}^{2}}{8 \pi r^{4}}
$$

where $G m_{p}^{2} /\left(8 \pi r^{4}\right)$ will find the sum of P chambers of the entire universe to obtain vacuum energy density.

The excited energy density is

$$
\rho_{u}=\frac{G m_{P} m_{s}}{4 \pi r^{4}}\left(1+\frac{m_{s}}{2 m_{P}}\right) \approx \frac{G m_{P} m_{s}}{4 \pi r^{4}} .
$$

The excited energy is

$$
\begin{aligned}
& \mathcal{E}_{r r}^{T}\left(m_{s}\right)=-\frac{r_{P}^{2} m_{s}}{r^{2} m_{P}}, \\
& u_{r}^{T}\left(m_{s}\right)=\frac{r_{P}^{2}}{r} \frac{m_{s}}{m_{P}} .
\end{aligned}
$$

(2) The interaction between excited energy flow and vacuum background

Vacuum has pressure on the excited energy $E_{u}\left(\rho_{u}\right)$, trying hard to press it into a certain $\mathrm{P}$ chamber and become $\mathrm{E}_{\mathrm{s}}$. As shown in figure 4 , the compressive force generated by 
spherical layer $\left(r_{a}-r_{b}\right)$ under the action of vacuum is

$$
\begin{gathered}
F_{u}\left(r_{a}-r_{b}\right)=\int_{r_{a}}^{r_{b}}-\frac{G m_{P}}{r_{P}^{2}} \cdot \frac{G m_{P} m_{s}}{4 \pi r^{4} c^{2}} \cdot 4 \pi r^{2} d r=-m_{s} c^{2}\left(\frac{1}{r_{a}}-\frac{1}{r_{b}}\right) \\
F_{u}(r-\infty)=-\frac{m_{s} c^{2}}{r} .
\end{gathered}
$$

Stress

$$
\sigma_{r}^{r}=-\frac{m_{s} c^{2}}{r} \cdot\left(4 \pi r^{2}\right)^{-1}=-\frac{m_{s} c^{2}}{4 \pi r^{3}}
$$

The spherically symmetric stress tensor [10]

$$
\sigma_{v}^{\mu}(u)=\operatorname{diag}\left[-\frac{m_{s} c^{2}}{4 \pi r^{3}}, \frac{m_{s} c^{2}}{8 \pi r^{3}}, \frac{m_{s} c^{2}}{8 \pi r^{3}}, \frac{-m_{s} c^{2} r_{P}}{4 \pi r^{4}}\right]=T_{v}^{\mu}(u) .
$$

When vacuum background gives the excited energy flow $\sigma_{v}^{\mu}(u)$, reaction is obtained at the same time.

$$
\sigma_{v}^{\mu}(B)=\operatorname{diag}\left[\frac{m_{s} c^{2}}{4 \pi r^{3}}, \frac{-m_{s} c^{2}}{8 \pi r^{3}}, \frac{-m_{s} c^{2}}{8 \pi r^{3}}, \frac{-m_{s} c^{2} r_{P}}{4 \pi r^{4}}\right]=T_{v}^{\mu}(B)
$$

From Hooke's equation we have

$$
\varepsilon_{v}^{\mu}(B)=E_{e}^{-1} \sigma_{v}^{\mu}(B)=\operatorname{diag}\left[\frac{r_{P}^{2} G m_{s}}{c^{2} r^{3}},-\frac{r_{P}^{2} G m_{s}}{2 c^{2} r^{3}},-\frac{r_{P}^{2} G m_{s}}{2 c^{2} r^{3}},-\frac{r_{P}^{2} G m_{s} r_{P}}{c^{2} r^{4}}\right]
$$

\subsection{Curvature of the P Chamber}

(1) Image

Under the reaction of the excited energy flow, the vacuum background $\mathrm{P}$ chamber is stretched along the $\hat{r}_{\text {direction and }}$ contracted transversely, as shown in figures 5, 6, and 7 .

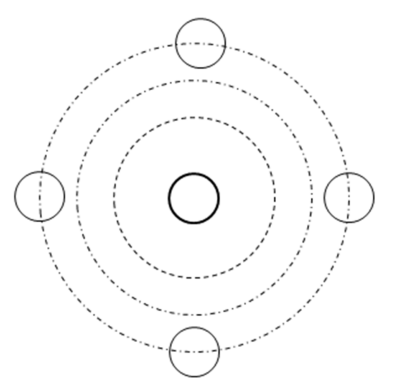

Figure 5. Vacuum state spherical P chamber.

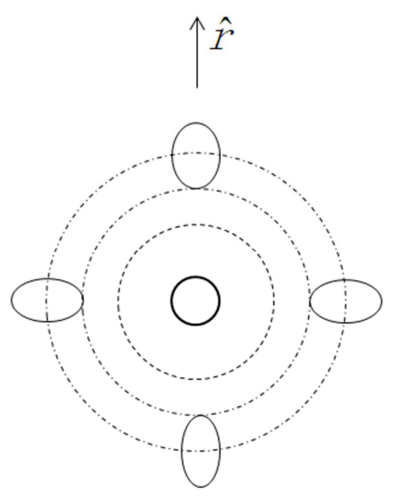

Figure 6. Excited ellipsoidal P chamber.

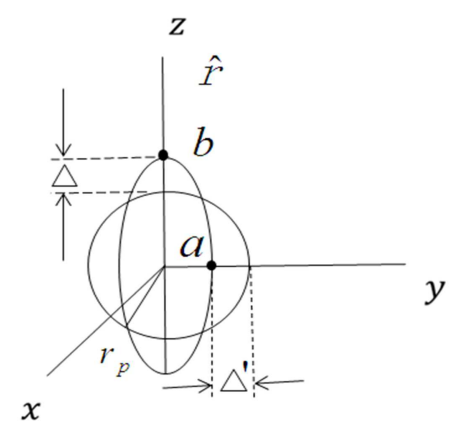

Figure 7. Ellipse.

(2) The curvature tensor

As shown in figure 7 , the linear curvature at point $b$ of ellipse is

$$
c_{b}=\frac{b}{a^{2}}=\frac{r_{P}+\Delta}{\left(r_{P}+\Delta^{\prime}\right)^{2}}=\frac{1}{r_{P}}\left(1+\frac{\Delta}{r_{P}}\right),
$$

where $\Delta=\varepsilon_{r}^{r}(B) r_{P}, \quad \Delta^{\prime}=0 \quad\left(u_{\theta}=u_{\phi}=0\right)$.

The surface curvature at point $b$ of the ellipsoid is

$$
c_{b}^{2}=\frac{1}{r_{P}^{2}}\left(1+\frac{\Delta}{r_{P}}\right)^{2} \cong \frac{1}{r_{P}^{2}}\left(1+2 \frac{\Delta}{r_{P}}\right)=\frac{1}{r_{P}^{2}}+\frac{2 \Delta}{r_{P}^{3}} .
$$

The surface curvature increment at point $b$ is

$$
c_{b}^{2}-\frac{1}{r_{P}^{2}}=\frac{2 \Delta}{r_{P}^{3}} .
$$


The $\hat{r}_{\text {directed curvature increment is }}$

$$
\left(c_{b}^{2}-\frac{1}{r_{P}^{2}}\right)=\frac{2 \Delta}{r_{P}^{3}}=\frac{2 \varepsilon_{r}^{r}(B)}{r_{P}^{2}}=\frac{2 G m}{c^{2} r^{3}} .
$$

It is usually considered that the space- time of vacuum is flat. If so, the 3-D spatial curvature $\left(R_{r}^{r}\right)_{a}[11]$ is in effect $\mathrm{P}$

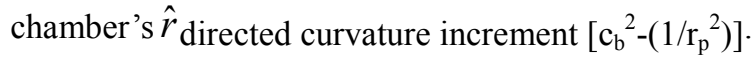

$$
\left(R_{r}^{r}\right)_{a}=\left(c_{b}^{2}-\frac{1}{r_{P}^{2}}\right)=\frac{2 \Delta}{r_{P}^{3}}=\frac{2 \varepsilon_{r}^{r}(B)}{r_{P}^{2}}=\frac{2 G m}{c^{2} r^{3}} .
$$

This is exactly Schwarzschild's solution for $\left(R_{r}^{r}\right)_{a}$, but we give the clear elasticity image on the curvature.

\subsection{Elastic Statement of Gravitation Equation}

From Eq. (38) and Hooke's equation we have

$$
\begin{gathered}
\sum_{r}^{r}(\text { curvature }) \equiv\left(c_{b}^{2}-\frac{1}{r_{p}^{2}}\right)\left(\frac{2}{r_{p}^{2}}\right)^{-1}=\left(R_{r}^{r}\right)_{a}\left(\frac{2}{r_{p}^{2}}\right)^{-1}= \\
\varepsilon_{r}^{r}(B)=E_{e}^{-1}\left(T_{r}^{r}(B)\right)_{a} . \\
\left(R_{r}^{r}\right)_{a}=\frac{2}{r_{P}^{2}} E_{e}^{-1}\left(T_{r}^{r}(B)\right)_{a}=\frac{8 \pi G}{c^{4}}\left(T_{r}^{r}(B)\right)_{a}
\end{gathered}
$$

Similarly, we have

$$
\begin{aligned}
& \left(R_{\theta}^{\theta}\right)_{a}=\frac{8 \pi G}{c^{4}}\left(T_{\theta}^{\theta}(B)\right)_{a}, \\
& \left(R_{\phi}^{\phi}\right)_{a}=\frac{8 \pi G}{c^{4}}\left(T_{\phi}^{\phi}(B)\right)_{a} .
\end{aligned}
$$

The static spherically symmetric3-D curvature equations are

$$
\left(R_{i}^{i}\right)_{a}=\frac{8 \pi G}{c^{4}}\left(T_{i}^{i}(B)\right)_{a} \quad i=1,2,3 .
$$

The non-spherically symmetric 4-D ordinary case should be

$$
G_{v}^{\mu}=\frac{8 \pi G}{c^{4}} T_{v}^{\mu}(B), \quad \mu, v=1,2,3,4 .
$$

There should be $G_{v ; \mu}^{\mu}=0$ on the left side of equations (45) as $T_{v ; \mu}^{\mu}=0$ on the right. For this reason, there must be $G_{v}^{\mu}=R_{v}^{\mu}-\frac{1}{2} \delta_{v}^{\mu} R$. Ordinarily curvature equations are

$$
\begin{gathered}
R_{v}^{\mu}-\frac{1}{2} \delta_{v}^{\mu} R=\frac{8 \pi G}{c^{4}} T_{v}^{\mu}(B), \\
R_{\mu v}-\frac{1}{2} g_{\mu v} R=\frac{8 \pi G}{c^{4}} T_{\mu v}(B) .
\end{gathered}
$$

It should be emphasized that $T_{\mu}$ in Einstein's equations is of field source, of $E_{s}$ and forced on it by conjecture while $T_{\mu \nu}(B)$ in equations (47) is $\mathrm{E}_{\mathrm{u}}$ 's and continuously distributed in and outside the "source" and an inevitable result of the elastomer's Hooke equation. Einstein's equations are only Hook's equations of the universal lump in linear approximation.

\subsection{Correlation Between Elastic Statement, Geometric Statement and Field Statement}

Geometric statement

$$
\begin{gathered}
g_{r r}=\left(1+\frac{2 G m_{s}}{c^{2} r}\right)=\left(1+\frac{2 u_{r}^{T}\left(m_{s}\right)}{r_{P}}\right), \\
g_{44}=\left(1-\frac{2 G m_{s}}{c^{2} r}\right)=\left(1-\frac{2 u_{r}^{T}\left(m_{s}\right)}{r_{P}}\right), \\
\Gamma_{r r}^{r}=-\frac{G m_{s}}{c^{2} r^{2}}=\frac{\varepsilon_{r r}^{T}\left(m_{s}\right)}{r_{p}}, \\
\left(\mathrm{R}_{r}^{r}\right)_{a}=\frac{2 G m_{s}}{c^{2} r^{3}}=\frac{2 \varepsilon_{r}^{r}(B)}{r_{p}^{2}}, \\
R=\frac{8 \pi G}{c^{4}} \rho_{u}\left(m_{s}\right) .
\end{gathered}
$$

Field Statement

$$
\begin{gathered}
c^{2}=\frac{G m_{P}}{r_{P}}, \\
\varphi=-\frac{G m_{s}}{r}=-\frac{c^{2} u_{r}^{T}\left(m_{s}\right)}{r_{P}}, \\
\Phi=c^{2}-\frac{G m_{s}}{r}=\frac{G m_{P}}{\left(r_{P}+u_{r}^{T}\left(m_{s}\right)\right)}=\frac{G m_{P}}{r_{P}}\left(1-\frac{u_{r}^{T}\left(m_{s}\right)}{r_{P}}\right) \\
\vec{E}=\frac{-G m_{s}}{r^{2}} \hat{r}=\frac{G m_{p}}{r_{p}^{2}} \varepsilon_{r r}^{T}\left(m_{s}\right) \hat{r} .
\end{gathered}
$$

\subsection{Origin of the Newtonian Gravitation}

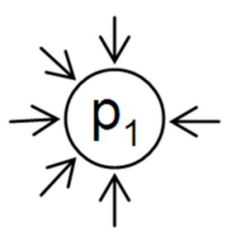

Weakened region

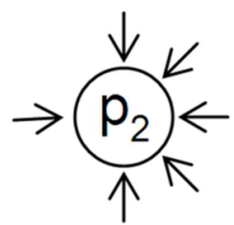

Figure 8. Deformation of the universal lump at a certain point in the weakened region has to bend both toward $P_{1}$ and $P_{2}$, hence mutual weakening. 


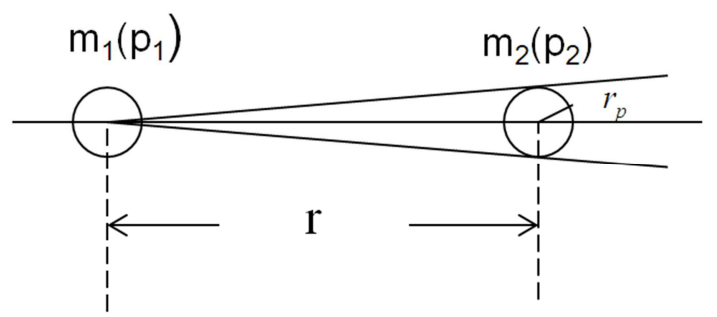

Figure 9. Particle equivalent cross section is $4 \pi r_{P}^{2}$, the corresponding solid angle is $\Omega=\frac{4 \pi r_{P}^{2}}{r^{2}}$.

$$
F_{u}(1)=\frac{G m_{P} m_{1}}{r_{P}^{2}}
$$

The universal continuum total surface force borne by the $\mathrm{P}$ chamber's outer surface of the isolated particle 1 . The resultant is now zero.

$$
\frac{G m_{P} m_{1}}{r_{P}^{2}} \cdot \frac{1}{4 \pi}
$$

The universal continuum's force on the surface of $P_{1}$ chamber in unit solid angle.

$$
\frac{G m_{P} m_{1}}{r_{P}^{2}} \cdot \frac{1}{4 \pi} \cdot \frac{4 \pi r_{P}^{2}}{r^{2}}
$$

The universal continuum's force on the surface of $\mathrm{P}_{1}$ chamber in the solid angle $4 \pi r_{p}{ }^{2} / r^{2}$ formed by $\mathrm{P}_{2}$ chamber.

$$
\frac{G m_{P} m_{1}}{r_{P}^{2}} \cdot \frac{1}{4 \pi} \cdot \frac{4 \pi r_{P}^{2}}{r^{2}} \cdot \frac{m_{2}}{m_{P}}=\frac{G m_{1} m_{2}}{r^{2}}
$$

The cut down amount of the force borne by $\mathrm{P}_{1}$ chamber surface via weakening by $\mathrm{m}_{2}$ at $\mathrm{r}$ in the solid angle $4 \pi r_{p}{ }^{2} / r^{2}$, the cut down rate of $m_{2}$ is $m_{2} / m_{p}$.

The Newtonian gravitation $\mathrm{F}_{\mathrm{G}}$ :

For isolated particle, the resultant force of universal continuum on $\mathrm{P}$ chamber is zero. When there are two particles, because of mutual weakening, the resultant is not zero but expressed as $\frac{G m_{1} m_{2}}{r^{2}} \hat{r},-\frac{G m_{1} m_{2}}{r^{2}} \hat{r}$, respectively. The Newtonian gravitation $\mathrm{F}_{\mathrm{G}}$ is the result of destruction of $\mathrm{F}_{\mathrm{u}}$ spherical symmetry, $-\frac{G m_{1} m_{2}}{r}$ is reduction in potential energy $E_{u}=\left(m_{1}+m_{2}\right) c^{2}, \frac{G m^{2}}{8 \pi r^{4}}$ only is a part of $\rho_{u}=\mathrm{G} \frac{m_{p} m}{4 \pi r^{4}}$ $+\mathrm{G} \frac{m^{2}}{8 \pi r^{4}}$.

\section{4. $Q$ Field in P Chamber Network}

\subsection{C sphere's Mechanical System}

The surface forces $\mathrm{F}_{\mathrm{u}}$ and $\mathrm{F}_{\mathrm{q}}$ in equilibrium constitute a $\mathrm{C}$ sphere as shown in figure 10 .

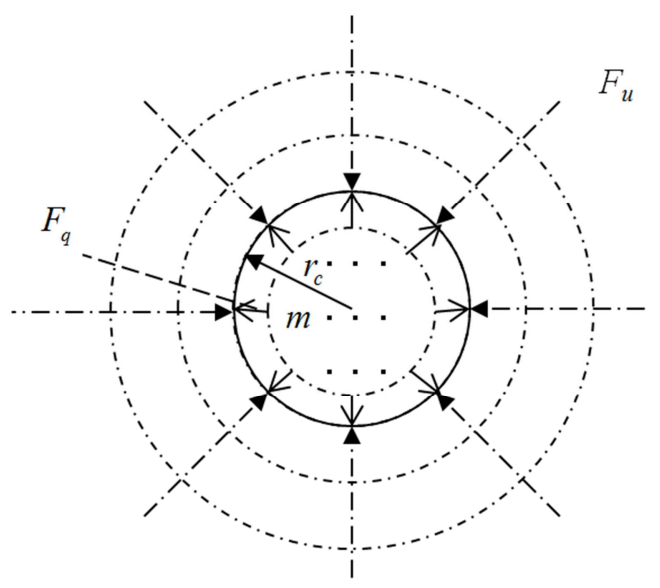

Figure 10. C sphere acted on by surface forces.

On the interface of the C sphere [8], $-\mathrm{mc}^{2} / \mathrm{r}_{\mathrm{c}}+\hbar \mathrm{c} / \mathrm{r}_{\mathrm{c}}{ }^{2}=0$, the particle is captivated in the $\mathrm{C}$ sphere jumping probabilistically. The $\mathrm{C}$ sphere and surrounding universal continuum constitute a mechanical system. The mass of $\mathrm{C}$ sphere is $\mathrm{m}$, its radius is $r_{c}=\hbar /(m c), \quad E_{s}$ distributed in the $P$ chamber jumping probabilistically, i.e. in the $\mathrm{C}$ sphere, $\mathrm{E}_{\mathrm{u}}$ distributed in the universal continuum in and outside the $\mathrm{C}$ sphere. The excited energy $E_{T}=E_{u}+E_{s}$ makes the system deviate from the vacuum state and generate the quantum field $\psi$, or Q field for short. Its relative change is $\Sigma_{c} \equiv \psi /\left|\psi_{v}\right|,\left|\psi_{v}\right|$ is the mode of the vacuum state function that is a constant. The generalized coordinate of the system is $q_{c}=r_{c} \psi /\left|\psi_{v}\right|$

Let's turn back to $\mathrm{P}$ chamber acted on by force $\mathrm{F}_{\mathrm{p}}=$ $-\left(\mathrm{Gm}_{\mathrm{p}}{ }^{2} / \mathrm{r}_{\mathrm{p}}{ }^{2}\right)\left[\left(\mathrm{E}_{\mathrm{u}}-\mathrm{E}_{\mathrm{s}}\right) / \mathrm{E}_{\mathrm{p}}\right]$, where $\mathrm{Gm}_{\mathrm{p}}{ }^{2} / \mathrm{r}_{\mathrm{p}}{ }^{2}$ is the single-side value of the vacuum state $P$ chamber interfacial equilibrium force. $\left(E_{\mathrm{u}}-\mathrm{E}_{\mathrm{s}}\right) / \mathrm{E}_{\mathrm{p}}=\Sigma_{\mathrm{p}}$, Similarly, for $\mathrm{C}$ sphere $\psi$ we have

$$
F_{c}=\frac{-\hbar c}{r_{c}^{2}} \frac{\psi}{\left|\psi_{V}\right|}
$$

where $\frac{\hbar c}{r_{c}{ }^{2}}$ is the single -side value of $\mathrm{C}$ sphere equilibrium force, $\frac{\psi}{\left|\psi_{v}\right|}=\Sigma_{c}$.

\subsection{The Equation of Motion}

The equation of motion of $\psi$ is

$$
\begin{gathered}
m r_{c} \frac{d^{2}}{d \tau^{2}} \psi /\left|\psi_{V}\right|=-\frac{\hbar c}{r_{c}^{2}} \psi /\left|\psi_{V}\right|, \\
\text { or } \frac{d^{2} \psi}{d \tau^{2}}=-\omega^{2} \psi, \quad \omega^{2}=\frac{m^{2} c^{4}}{\hbar^{2}} .
\end{gathered}
$$

Lagrangian functions is

$$
L_{c}=\frac{1}{2} m \dot{\bar{q}}_{c} q_{c}-\frac{1}{2} m \omega^{2} \bar{q}_{c} q_{c} .
$$

Further, 


$$
\begin{aligned}
& \frac{d^{2}}{d \tau^{2}} \psi_{\phi}=-\frac{m^{2} c^{4}}{\hbar^{2}} \psi_{\phi}, \\
& \frac{d}{d \tau} \psi_{D}=-i \frac{m c^{2}}{\hbar} \psi_{D} .
\end{aligned}
$$

Where $\psi_{\phi}, \psi_{D}$ are the state functions of the scalar particle and that of Dirac particle, respectively.

The field equations are

$$
\begin{gathered}
\gamma_{\mu} \partial_{\mu} \psi_{D}=-\frac{m c}{\hbar} \psi_{D}, \\
\gamma_{\mu}^{2} \partial_{\mu}^{2} \psi_{\phi}=\frac{m^{2} c^{2}}{\hbar^{2}} \psi_{\phi},
\end{gathered}
$$

respectively.

\subsection{Puzzlers of the Quantum Theory}

\section{(1) Material basis of $\psi$}

The wave function $\psi$ is the joint effect of $\mathrm{P}$ net and universal continuum in $\mathrm{E}_{\mathrm{u}} \leftrightarrow \mathrm{E}_{\mathrm{s}}$ oscillation. The wave nature material basis is universal continuum, particle nature material basis is $\mathrm{P}$ chamber, probabilistic nature material basis is the high frequency transformation $\mathrm{E}_{\mathrm{u}} \leftrightarrow \mathrm{E}_{\mathrm{s}}$.

(2) Connection of quantum theory and gravitational theory

The free particle wave function can be represented by the physical quantities $\rho_{\mathrm{u}}, \mathrm{R}$ and $\varepsilon$ in the $U$ field [12].

$$
R=\frac{8 \pi G}{c^{4}} \rho_{u}, \rho_{u}=\frac{1}{2} E_{e} \varepsilon^{2} .
$$

The scalar field

$$
\begin{gathered}
\phi=A_{\phi} \exp \left[i k_{\mu} x_{\mu}\right], \\
A_{\phi}=\frac{\hbar c}{\sqrt{2 E V}}=\left(\frac{\hbar c}{16 \pi} \frac{r_{c}^{2}}{r_{P}^{2}}\right)^{1 / 2} R^{1 / 2}=\frac{c^{2}}{\sqrt{G}} \frac{1}{\sqrt{16 \pi}} \frac{r_{c}}{r_{p}} \varepsilon \\
\phi^{*} \phi=\frac{\hbar c}{16 \pi} \frac{r_{c}^{2}}{r_{P}^{2}} R=\rho_{u}\left[\frac{1}{2} r_{c}^{2}\right], \\
\frac{8 \pi}{R} \cdot \phi^{*} \phi \frac{2 m}{\hbar^{2}} \cdot m c^{2}=\frac{c^{4}}{G} .
\end{gathered}
$$

The Dirac field

$$
\begin{gathered}
\psi=A_{D} u_{\vec{k}, \bar{\sigma}} \exp \left[i k_{\mu} x_{\mu}\right], \\
A_{D}=\frac{1}{\sqrt{V}} \sqrt{\frac{m c^{2}}{E}}=\left(\frac{1}{8 \pi} \frac{r_{c}}{r_{P}^{2}}\right)^{1 / 2} R^{1 / 2}=\left(\frac{r_{c}}{8 \pi r_{p}^{4}}\right)^{1 / 2} \varepsilon \\
\bar{\psi} \psi=\frac{1}{8 \pi} \frac{r_{c}}{r_{P}^{2}} R=\rho_{u}\left[\frac{r_{c}}{\hbar c}\right]
\end{gathered}
$$

$$
\frac{8 \pi}{R} \cdot \bar{\psi} \psi \cdot m c^{2}=\frac{c^{4}}{G}
$$

The wave function is the wave function of various special field, is also the strain (stress) wave function. The electromagnetic wave must have an adjoint strain wave.

(3) Quantum entanglement

Electron 1 and electron 2 constitute the quantum state $\mid \psi>=\frac{1}{\sqrt{2}}\left[\left|\uparrow>_{1}\right| \downarrow>_{2}-\left|\downarrow>_{1}\right| \uparrow>_{2}\right]$ with zero spin, whose physical essence is, by means of action, to enable two electrons to possess a common $\mathrm{E}_{\mathrm{u}}$, in collaboration the common $\mathrm{E}_{\mathrm{u}}$ with $\mathrm{E}_{\mathrm{s}}$ (1) and $\mathrm{E}_{\mathrm{s}}$ (2) carries out $\mathrm{E}_{\mathrm{u}} \leftrightarrow \mathrm{E}_{\mathrm{s}}$ oscillation synchronously. $|\uparrow>,| \downarrow>$ randomly assigned but ensuring conservation of angular momentum. When the two electrons in entangled state are set far apart, despite the absence of conventional mechanical action, they can still be entangled via their $\mathrm{E}_{\mathrm{u}}$ in common (not Newtonian gravitation). If $|\uparrow\rangle$ is found here, then $|\downarrow\rangle$ will certainly be found far away. This is no instantaneous action but the fact that the $E_{u}$ common to $P_{1}$ and $p_{2}$ has all along had them coupled. That is Feyman s expectation [12].

(4) Uncertainty relation of the energy

If we measure $\mathrm{E}_{\mathrm{s}}$ by means of $\mathrm{s}$, we are not able to find the instantaneous value but just the average value in an interval $\Delta \mathrm{t}:$

$$
E_{s}=\frac{1}{\Delta t} \int_{o}^{\Delta t} \frac{E_{T}}{2}\left(1+\cos \omega_{P} t\right) d t=\frac{E_{T}}{2}+\frac{E_{T}}{2} \frac{\sin \omega_{P} \Delta t}{\Delta t \omega_{P}}
$$

The true vacuum is the dynamic equilibrium state, $\mathrm{E}_{\mathrm{P}}$ enters into the energy transformation. Where $E_{T}=2 \hbar\left(\omega_{P}+\omega\right)$.

The deviation is

$$
E_{s}-\bar{E}_{s}=E_{s}-\frac{E_{T}}{2}=\frac{E_{T}}{2} \frac{\sin \omega_{P} \Delta t}{\Delta t \omega_{P}}
$$

Further,

$$
\begin{gathered}
\left(E_{s}-\overline{E_{s}}\right)^{2}=\frac{E_{T}^{2}\left(\sin \omega_{p} \Delta t\right)^{2}}{4(\Delta t)^{2}\left(\omega_{p}\right)^{2}}, \\
\overline{\left(E_{s}-\bar{E}_{s}\right)^{2}}=\frac{E_{T}^{2}}{4} \frac{\frac{1}{2}}{\Delta t^{2} \omega_{P}^{2}} \doteq \frac{\hbar^{2}}{2 \Delta t^{2}}, \\
\sqrt{\overline{\left(E_{s}-\bar{E}_{s}\right)^{2}}} \equiv \Delta E=\frac{\hbar}{\sqrt{2} \Delta t} .
\end{gathered}
$$

From Eq. (77), we obtain the uncertainty relation

$$
\Delta E \Delta t=\frac{\hbar}{\sqrt{2}} .
$$

The energy is uncertain in measurement but conservative in $\mathrm{E}_{\mathrm{T}}=\mathrm{E}_{\mathrm{u}}+\mathrm{E}_{\mathrm{s}}$. 


\section{Universe Sphere Kinetics}

\subsection{Mechanical System of Universal Sphere}

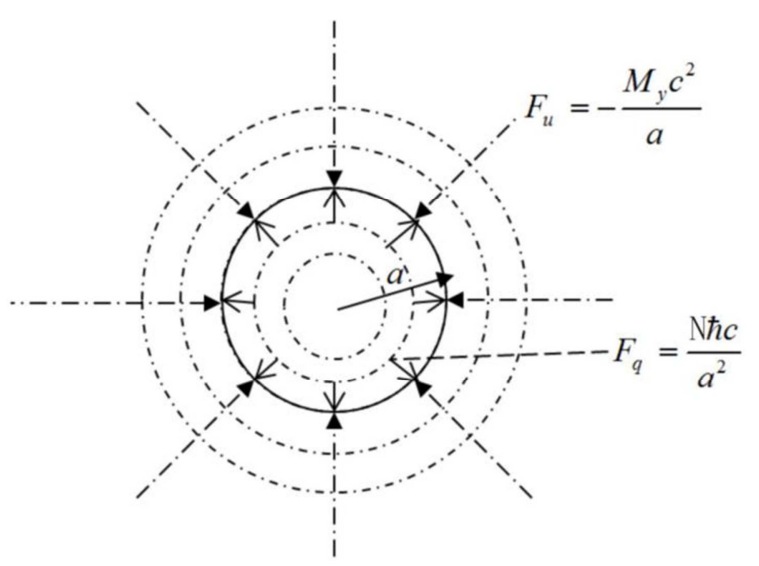

Figure 11. Universal sphere acted on by surface forces.

The "initial" universe is composed of vacuum plus excited state universal sphere as shown in Figure 11, of mass $M_{y}$, radius $a . \mathrm{E}_{\mathrm{u}}$ is distributed in and out of the sphere. $\rho_{\mathrm{u}}$ is homogenously distributed inside the sphere while outside, $\rho_{u}=\left(G M_{y} m_{p}\right) /\left(4 \pi r^{4}\right) . E_{s}$ is homogenously distributed inside the universal sphere. $\mathrm{E}_{\mathrm{s}}=\mathrm{E}_{\phi}+\mathrm{E}_{\sigma}+\mathrm{E}\left(a^{*}\right), \mathrm{E}_{\phi}$ is the (dark) energy of $\mathrm{N} \phi$ field particles, $\mathrm{E}_{\sigma}$ is the (visible and invisible) energy of ordinary materials, while $\mathrm{E}\left(a^{*}\right)$ is the expanded or contracted energy of the universal sphere as shown in figure 11.

As shown in figure 11, the interface exterior bears the universal compressive surface force $F_{u}=-M_{y} c^{2} / a$; while the interior of the interface bears the $\phi$ field quantum dispersive surface force $\mathrm{F}_{\mathrm{q}}=\mathrm{Nhc} / \mathrm{a}^{2}$.

When equilibrium,

$$
\frac{N \hbar c}{a_{0}^{2}}=\frac{M_{y} c^{2}}{a_{0}}
$$

where $a_{0}$ is the radius of the universal sphere in equilibrium state. From Eq. (79) we can obtain $N \hbar c=M_{y} c^{2} a_{0}$. For the $P$ sphere vacuum equilibrium state there is $\mathrm{Gm}_{\mathrm{p}} / \mathrm{r}_{\mathrm{p}}=\mathrm{c}^{2}$. Similarly, for the universal sphere equilibrium state there is also $\mathrm{GM}_{\mathrm{y}} / \mathrm{a}_{0}=\mathrm{c}^{2}$ that can lead to $\mathrm{GM}_{\mathrm{y}}{ }^{2}=\mathrm{M}_{\mathrm{y}} \mathrm{c}^{2} \mathrm{a}_{0}$. The total force borne by the universal sphere's interface is

$$
F_{\mathrm{T}} \equiv F_{q}+F_{u}=\frac{N \hbar c}{a^{2}}-\frac{M_{y} c^{2}}{a}=-M_{y} c^{2}\left(\frac{a-a_{0}}{a^{2}}\right) \approx-M_{y} c^{2}\left(\frac{a-a_{0}-a_{P}}{a^{2}}\right) .
$$

Let $\mathrm{q}_{\mathrm{y}}=\mathrm{a}_{0}\left[\left(\mathrm{a}-\mathrm{a}_{0}-\mathrm{a}_{\mathrm{p}}\right) / \mathrm{a}_{0}\right]$, where $\mathrm{a}_{\mathrm{p}}$ is the universal sphere radius when stable vacuum is being formerly formed, $\mathrm{a}_{\mathrm{p}}<<\mathrm{a}_{0}$. In our study of the universe, we simply proceed from basic forces without using R-W metric and Einstein's gravitational equations [13].

\subsection{Equation of Motion}

In the extraspherical flat coordinate system, time is not related to $a$ and the equation of motion is

$$
M_{y} a_{0} \frac{d^{2}}{d t^{2}}\left(\frac{a-a_{0}-a_{P}}{a_{0}}\right)=-M_{y} c^{2}\left(\frac{a-a_{0}-a_{P}}{a^{2}}\right) .
$$

In the intraspherical coordinate system, 3-D space is flat, time is related to $a[14], d t_{a} \equiv \frac{a_{0}}{a} d t$, the equation of motion is

$$
M_{y} a_{0} \frac{d^{2}}{d t_{a}^{2}}\left(\frac{a-a_{0}-a_{P}}{a_{0}}\right)=-\frac{M_{y} c^{2}}{a_{0}}\left(\frac{a-a_{0}-a_{P}}{a_{0}}\right)=-M_{y} \omega_{y}^{2}\left(a-a_{0}-a_{P}\right),
$$

Where $\omega_{y}=\frac{c}{a_{0}}$.

The forces we are aware of in the universe sphere are

$$
\begin{aligned}
& F_{u}^{a}=-\frac{M_{y} c^{2}}{a_{0}} \frac{\left(a-a_{P}\right)}{a_{0}}=-\frac{c^{4}}{G} \frac{\left(a-a_{P}\right)}{a_{0}}, \\
& F_{q}^{a}=\frac{M_{y} c^{2}}{a_{0}}=\frac{c^{4}}{G}=\frac{G m_{p}^{2}}{r_{p}^{2}}=\frac{\hbar c}{r_{p}^{2}}=\frac{N \hbar c}{a_{0}^{2}} .
\end{aligned}
$$

There are the similar basic actions in Einstein's equations for the horizon [4].

The equation of motion can be simplified as

$$
\frac{d^{2}}{d t_{a}^{2}}\left(a-a_{0}-a_{p}\right)=-\omega_{y}^{2}\left(a-a_{0}-a_{p}\right)
$$

Whose solution leads to

$$
\begin{gathered}
a=a_{0}\left(1-\cos \omega_{y} t_{a}\right)+a_{p}, \\
\frac{d a}{d t_{a}} \equiv a^{*}=\operatorname{csin} \omega_{y} t_{a} \\
\frac{d^{2} a}{d t_{a}^{2}} \equiv a^{* *}=\omega_{y}^{2} a_{0} \cos \omega_{y} t_{a}=\frac{c^{2}}{a_{0}} \cos \omega_{y} t_{a}=-\omega_{y}^{2}\left(a-a_{0}-a_{p}\right) .
\end{gathered}
$$

Lagrangian function is

$$
L_{y}=\frac{1}{2} M_{y}\left(q_{y}^{*}\right)^{2}-\frac{1}{2} M_{y} \omega_{y}^{2} q_{y}^{2}-\frac{1}{2} M_{y} c^{2} .
$$




\subsection{Friedmann Equation}

From equations (87) and (86) we have

$$
\left(a^{*}\right)^{2}=c^{2}\left[1-\left(\frac{a-a_{0}-a_{p}}{a_{0}}\right)^{2}\right] .
$$

Using $, \dot{a} \equiv \frac{d a}{d t},\left(\frac{d a}{d t_{a}}\right)^{2}=(\dot{a})^{2}\left(\frac{a}{a_{0}}\right)^{2}, \frac{G M_{y}}{a_{0}}=c^{2}$ and

$$
\rho_{s}=\frac{M_{y} c^{2}}{\frac{4 \pi}{3} a^{3}}
$$

Further we have

$$
\begin{gathered}
\frac{\left(a^{*}\right)^{2}}{c^{2} a^{2}}+\frac{1}{a^{2}}=\frac{8 \pi G}{3 c^{4}} \rho_{S}\left[-\frac{a^{3}}{2 a_{0}^{3}}+\frac{a^{2} a_{p}}{a_{0}^{3}}-\frac{a a_{p}^{2}}{2 a_{0}^{3}}+\frac{a^{2}}{a_{0}^{2}}-\frac{a a_{p}}{a_{0}^{2}}+\frac{a}{2 a_{0}}\right], \\
\frac{(\dot{a})^{2}}{c^{2} a^{2}}+\frac{1}{a^{2}}=\frac{8 \pi G}{3 c^{4}} \rho_{S}\left[1+\frac{a_{p}}{a_{0}}-\frac{a_{p}}{a}\left(1+\frac{a_{p}}{2 a_{0}}\right)\right]
\end{gathered}
$$

In the intraspherical coordinate system, when $a=a_{0}$ (equilibrium state), $a=2 a_{0}$ (static state),

$$
\frac{\left(a^{*}\right)^{2}}{c^{2} a^{2}}+\frac{1}{a^{2}}=\frac{8 \pi G}{3 c^{4}} \rho_{s} .
$$

In the extraspherical coordinate system, when $a \gg a_{p}$,

$$
\frac{(\dot{a})^{2}}{c^{2} a^{2}}+\frac{1}{a^{2}}=\frac{8 \pi G}{3 c^{4}} \rho_{s}
$$

The Eq. (94) and Eq. (95) are Friedmann's equation [16, 17], which is simply a special example of Eq. (92) and (93), respectively. The Eq. (92) can be rewritten as $\mathrm{E}_{\mathrm{u}}$ conservation equation:

$$
\frac{1}{2} M_{y}\left(a^{*}\right)^{2}+E_{u p}=M_{y} c^{2}=E_{u T},
$$

where

$$
E_{u P}=\left\{M_{y} c^{2}-\frac{G M_{y}^{2}}{a}\left[\left(\frac{a^{2}-a a_{P}}{a_{0}^{2}}\right)+\left(-\frac{a^{3}}{2 a_{0}^{3}}+\frac{a^{2} a_{P}}{a_{0}^{3}}-\frac{a a_{P}^{2}}{2 a_{0}^{3}}\right)\right]\right\} .
$$

\subsection{Dark Energy and Dark Matter}

(1) The principle of the work-energy

Performing integration on the two sides of Eq. (82),

$$
\begin{aligned}
& \int_{a_{P}}^{a} M_{y} \frac{d^{2} a}{d t_{a}^{2}} d a=\int_{a_{P}}^{a} \frac{M_{y} c^{2}}{a_{0}} d a-\int_{a_{P}}^{a} \frac{M_{y} c^{2}}{a_{0}^{2}}\left(a-a_{P}\right) d a, \\
& \int_{a_{p}}^{a} M_{y} \frac{d^{2} a}{d t_{a}^{2}} d a=\frac{1}{2} M_{y}\left[a^{*}(a)\right]^{2}-\frac{1}{2} M_{y}\left[a^{*}\left(a_{p}\right)\right]^{2},
\end{aligned}
$$

Equation (98) becomes

$$
\begin{aligned}
\frac{1}{2} \mathrm{M}_{\mathrm{y}}\left[a^{*}(a)\right]^{2}+\mathrm{E}_{\phi}(a)+\mathrm{E}_{\sigma}(a)=\frac{1}{2} \mathrm{M}_{\mathrm{y}}\left[a^{*}\left(a_{\mathrm{p}}\right)\right]^{2}+\mathrm{E}_{\phi}\left(a_{\mathrm{p}}\right)+\mathrm{E}_{\sigma}\left(a_{p}\right) \\
\mathrm{Q}=0.30, \mathrm{Q}^{2}=0.09, a=0.3 a_{0}
\end{aligned}
$$

Obviously $a^{*}\left(a_{p}\right)=0$, Letting $E_{\phi}\left(a_{p}\right)=M_{y} c^{2}, E_{\sigma}\left(a_{p}\right)=0$, $\mathrm{Q} \equiv\left(\frac{a-a_{p}}{a_{0}}\right)$, there will be the $\mathrm{E}_{\mathrm{s}}$ conservation equation:

$$
\frac{1}{2} M_{y}\left[a^{*}(a)\right]^{2}+E_{\phi}(a)+E_{\sigma}(a)=M_{y} c^{2}=E_{s T},
$$

where

$$
\begin{gathered}
E_{\phi}=M_{y} c^{2}(1-Q), \\
E_{\sigma}=M_{y} c^{2} \frac{1}{2} Q^{2}, \\
\mathrm{E}\left(a^{*}\right)=\frac{1}{2} M_{y}\left(a^{*}\right)^{2}=M_{y} c^{2}\left(Q-\frac{Q^{2}}{2}\right)
\end{gathered}
$$

$\mathrm{E}_{\phi}$ represents the so-called dark energy [33, 34], $\mathrm{E}_{\sigma}$ the energy of ordinary matter, $\mathrm{E}\left(a^{*}\right)$ the kinetic energy for expanding (or contracting) motion of universe, or the so-called energy of "dark matter", $\mathrm{E}_{\mathrm{ST}}$ total $\mathrm{E}_{\mathrm{s}}$.

(2) The proportions of all kinds of energies of current universe

If the percentage of the dark energy accounting for the total energy $M_{y} c^{2}$ is $\frac{E_{\phi}}{E_{T}}=1-Q=0.70$, then

$$
\begin{gathered}
\frac{E_{\sigma}}{E_{T}}=\frac{Q^{2}}{2}=0.045, \\
\frac{E\left(a^{*}\right)}{E_{T}}=\mathrm{Q}-\frac{Q^{2}}{2}=0.255 .
\end{gathered}
$$

From $a=0.3 \quad a_{0} \quad, \quad a=a_{0}\left(1-\cos \omega_{y} t_{a}\right), a^{*}=$ 
$c \sin \omega_{y} t_{a}, a^{* *}=\frac{c^{2}}{a_{0}} \cos \omega_{y} t_{a}$, we have $a^{*}=0.7 c, a^{* *}>$ 0 .

Our present-day universe: $a=0.3 a_{0}, a^{*}=0.7 \mathrm{c}$, $a^{* *}>0$ (accelerated expansion); Dark energy accounts for $70 \%$, ordinary matter accounts for $4.5 \%$, "dark matter" accounts for $25.5 \%$.

\subsection{Ly and $\mathcal{L}_{y}$}

$$
L_{y}=\frac{1}{2} M_{y}\left(a^{*}\right)^{2}-\left(\mathrm{E}_{\phi}+\mathrm{E}_{\sigma}\right) .
$$

By making use of $Q \equiv \frac{a-a_{p}}{a_{0}}$, Eq. (105) and Eq. (106), we have

$$
L_{y}=\frac{1}{2} M_{y} c^{2}\left(\frac{a_{0}^{2}}{c^{2}}\left(Q^{*}\right)^{2}\right)-\frac{1}{2} M_{y} c^{2}\left(Q^{2}-2 Q+1\right)-\frac{1}{2} M_{y} c^{2},
$$

where $Q^{*} \equiv \frac{d}{d t_{a}} Q$. Making use of $\frac{M_{y} c^{2}}{V}=\rho_{s} \dot{\sim} \rho_{u}=\frac{c^{4}}{8 \pi G} R$, we have

$$
\mathcal{L}_{y}=\frac{-c^{4}}{16 \pi G} R+\frac{C^{4}}{16 \pi G} R \frac{a_{0}^{2}}{c^{2}}\left(Q^{*}\right)^{2}-\frac{c^{4}}{16 \pi G} R\left(Q^{2}-2 Q+1\right) .
$$

Letting $\Phi \equiv \frac{c^{2}}{\sqrt{G}}\left(\frac{a-a_{0}-a_{P}}{a_{0}}\right)=\frac{c^{2}}{\sqrt{G}}(Q-1)=\frac{\sqrt{\hbar c}}{r_{P}}(Q-1)$ and $\Phi^{*} \equiv \frac{d}{d t_{a}} \Phi$, there will be

$$
\mathcal{L}_{y}=\frac{-C^{4}}{16 \pi G} R+\frac{a_{0}^{2}}{16 \pi c^{2}} R\left(\Phi^{*}\right)^{2}-\frac{1}{16 \pi} R \Phi^{2} .
$$

Letting $\varphi \equiv \frac{c^{2}}{\sqrt{G}}\left(\frac{a-a_{P}}{a_{0}}\right)=\frac{c^{2}}{\sqrt{G}} Q=\frac{\sqrt{\hbar c}}{r_{p}} Q \quad$ and $\varphi^{*} \equiv \frac{d}{d t_{a}} \varphi, \quad$ there will be

$$
\mathcal{L}_{y}=\frac{-C^{4}}{8 \pi G} R+\frac{a_{0}^{2}}{16 \pi c^{2}} R\left(\varphi^{*}\right)^{2}-\frac{1}{16 \pi} R \varphi^{2}+\frac{C^{2}}{8 \pi \sqrt{G}} R \varphi
$$

Letting $\phi \equiv \frac{c^{2}}{\sqrt{G}} Q=\frac{\sqrt{\hbar c}}{r_{P}} Q, \sigma \equiv \frac{c^{2}}{\sqrt{G}} Q^{2}=\frac{\sqrt{\hbar c}}{r_{P}} Q^{2}, \quad \phi^{*} \equiv \frac{d}{d t_{a}} \phi \quad$ and $\quad \sigma^{*} \equiv \frac{d}{d t_{a}} \sigma$, results in

$$
\mathcal{L}_{y}=\frac{-c^{4}}{8 \pi G} R+\frac{a_{0}{ }^{2}}{32 \pi c^{2}} R\left(\phi^{*}\right)^{2}+\frac{c^{2}}{8 \pi \sqrt{G}} R \phi+\frac{a_{0}{ }^{2}}{128 \pi \sqrt{G}} R \frac{\left(\sigma^{*}\right)^{2}}{\sigma}-\frac{c^{2}}{16 \pi \sqrt{G}} R \sigma
$$

For lack of the one and only method of determining $\mathcal{L}$, people have given out many $\mathcal{L}_{y}[18-22]$.

\section{Conclusion}

(1) Both $F_{q}$ and $F_{u}$ that are duality basic actions in universe [8] dominate the oscillation of three sphere. For further details see the table 1 .

(2) The duality framework, has founded the fundamental equations of the gravitation, quantum and universe by the unified simple method; includes the whole framework of the modern fundamental physics and can solve the puzzlers about the singularity, non-locality, uncertainty of energy, et al.

(3) The duality framework, can explain naturally many puzzlers of the quantum theory: the wave-particle duality, probabilistic nature, uncertainty of the energy and quantum entanglement; no using Einstein's theory, can obtain the mass- energy relation, Einstein's equations, Friedmann's equation, the acceleration of the universe expansion and the proportions of dark energy, "dark matter" and ordinary matter. The duality framework includes, links and foreruns both the gravitation theory and the quantum theory, and is their common material foundation to give their unified harmonious image.

(4) The $E_{u}$ and $E_{s}$ are transformed into each other at high frequency. The average effect of a long period is corresponding to $\mathrm{U}$ field and $\mathrm{Q}$ field. Space is the complex duality mechanical system of the $U$ field and $Q$ field. The excited $U$ field and $Q$ field simultaneously are two opposite faces of the particle. This opposite-unified entity is called the complex field and expressed as the direct product of $U$ field and $Q$ field. The gravitational field and its matching field are synchroquantized. This is our duality unity of gravitational field with other field. For further details see the table 2.

(5) Daring predictions: The electromagnetic wave must have an adjoint strain wave. The gravitational wave must have an adjoint wave of matching field [35]. Because the energy of "dark matter" is the kinetic energy for expanding (or contracting) motion of universe, it is in the wrong direction to find the particle of the "dark matter" [36-43]. 
Table 1. Three sphere oscillation

\begin{tabular}{|c|c|c|c|}
\hline Mechanical system & Psphere & C sphere & Y sphere \\
\hline Equilibrium state & $\frac{m_{P} c^{2}}{r_{P}}=\frac{\hbar c}{r_{P}^{2}} \quad r_{P}=\frac{\hbar}{m_{P} c}$ & $\frac{m c^{2}}{r_{c}}=\frac{\hbar c}{r_{c}^{2}} \quad r_{c}=\frac{\hbar}{m c}$ & $\frac{M_{y} \mathrm{c}^{2}}{a_{0}}=\frac{N \hbar c}{a_{0}^{2}} \quad a_{0}=\frac{N \hbar}{M_{y} c}=\frac{G M_{y}}{c^{2}}$ \\
\hline Generalized coordinate & $q_{P} \equiv r_{P} \Sigma_{P} \equiv r_{P}\left[\frac{E_{u}-E_{s}}{E_{P}}\right]$ & $q_{c} \equiv r_{c} \Sigma_{c} \equiv r_{c} \frac{\psi}{\left|\psi_{V}\right|}$ & $q_{y} \equiv a_{0} \Sigma_{y} \equiv a_{0}\left[\frac{a-a_{0}-a_{p}}{a_{0}}\right]$ \\
\hline Generalized action & $F_{P}=-\frac{m c^{2}}{r_{P}}+\frac{\hbar c}{r_{P}^{2}} \frac{m_{s}}{m_{P}} \equiv-\frac{G m_{P}^{2}}{r_{P}^{2}}\left[\frac{E_{u}-E_{s}}{E_{P}}\right]$ & $F_{c}=-\frac{G m_{P}^{2}}{r_{c}^{2}} \frac{\psi}{\left|\psi_{V}\right|}$ & $F_{y}=-\frac{M_{y} c^{2}}{a}+\frac{N \hbar c}{a^{2}}=-\frac{G M_{y}^{2}}{a_{0}^{2}}\left[\frac{a-a_{0}-a_{p}}{a^{2}}\right] a_{0}$ \\
\hline $\mathrm{L}$ & $L_{p}=\frac{1}{2} m_{P} \dot{q}_{P}^{2}-\frac{1}{2} m_{P} \omega_{P}^{2} q_{P}^{2}$ & $L_{c}=\frac{1}{2} m \dot{\bar{q}}_{c} \dot{q}_{c}-\frac{1}{2} m \omega^{2} \bar{q}_{c} q_{c}$ & $L_{y}=\frac{1}{2} M_{y}\left(q_{y}^{*}\right)^{2}-\frac{1}{2} M_{y} \omega_{y}^{2} q_{y}^{2}-\frac{1}{2} M_{y} c^{2}$ \\
\hline Equation of motion & $\begin{array}{l}m_{P} r_{P} \frac{d^{2}}{d \tau^{2}}\left[\frac{E_{u}-E_{s}}{E_{P}}\right]=-\frac{G m_{P}^{2}}{r_{P}^{2}}\left[\frac{E_{u}-E_{s}}{E_{P}}\right] \\
\frac{d^{2}}{d \tau^{2}}\left[\frac{E_{u}-E_{s}}{E_{P}}\right]=-\omega_{P}^{2}\left[\frac{E_{u}-E_{s}}{E_{P}}\right]\end{array}$ & $\begin{array}{l}m r_{c} \frac{d^{2}}{d \tau^{2}}\left[\frac{\psi}{\left|\psi_{V}\right|}\right]=-\frac{G m_{P}^{2}}{r_{c}^{2}}\left[\frac{\psi}{\left|\psi_{V}\right|}\right] \\
\frac{d^{2}}{d \tau^{2}} \psi=-\omega^{2} \psi\end{array}$ & $\begin{array}{l}M_{y} a_{0} \frac{d^{2}}{d t_{a}^{2}}\left[\frac{a-a_{0}-a_{P}}{a_{0}}\right]=-\frac{G M_{y}^{2}}{a_{0}^{2}}\left[\frac{a-a_{0}-a_{P}}{a_{0}}\right] \\
\frac{d^{2}}{d t_{a}^{2}}\left[\frac{a-a_{0}-a_{P}}{a_{0}}\right]=-\omega_{y}^{2}\left[\frac{a-a_{0}-a_{P}}{a_{0}}\right]\end{array}$ \\
\hline Solution & $\begin{array}{l}E_{u}=\frac{E_{T}}{2}\left(1-\cos \omega_{P} \tau\right) \\
E_{s}=\frac{E_{T}}{2}\left(1+\cos \omega_{P} \tau\right) \\
\text { oscillation of } E_{u} \leftrightarrow E_{s}\end{array}$ & $\begin{array}{l}\psi=\psi(\vec{r}) e^{-i \omega \tau} \\
\text { Oscillation of } \psi \text { effect }\end{array}$ & $\begin{array}{l}a=a_{0}\left(1-\cos \omega_{y} t_{a}\right)+a_{p} \\
\text { Oscillation of curvature effect } \\
\text { of Y sphere }\end{array}$ \\
\hline
\end{tabular}

Table 2. The complex field.

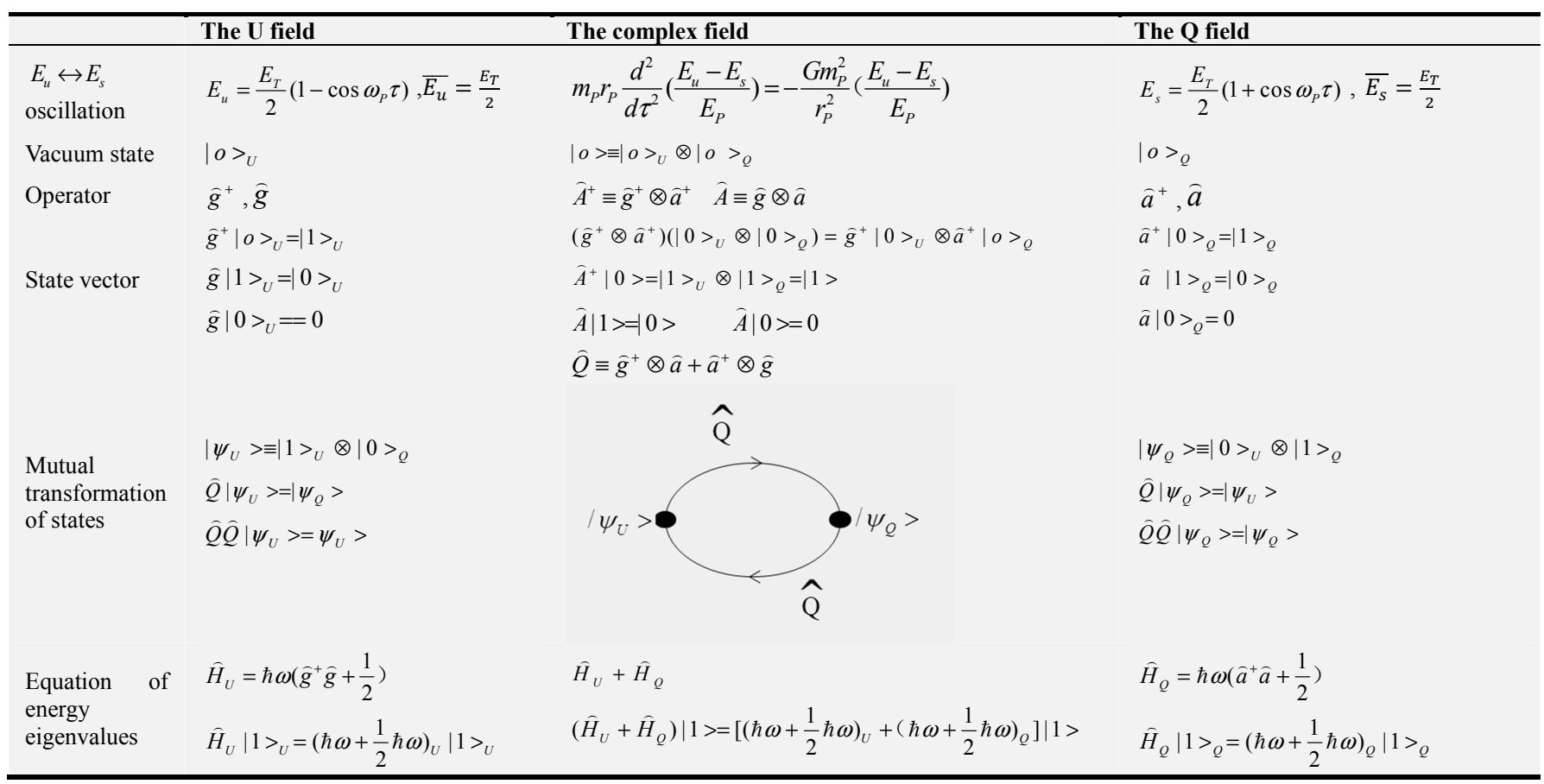

\section{References}

[1] Toma's Orti'n, Gravity and Strings (Cambridge University Press, 2004), p. 45-169.

[2] Thomas Thiemann, Modern Canonical Quantum General Relativity (Cambridge University Press, 2007), p. 400.

[3] Julius Wess and Jonathan Bagger, Supersymmetry and Supergravity, $2^{\text {nd }}$ (Princeton University Press 1992).

[4] T. Padmanabhan, Gravitation: Foundation and Frontiers
(Cambridge University Press and Peking University Press, 2013), P. 466, 474, 673.

[5] Shu-Jin Wang, "Planckon densely piled vacuum", American Journal of Modern Physics, Vol. 4, No. 1-1, 10-17 (2015).

[6] Amrit Sorli, et al. "Cosmology of Einstein's now", American Journal of Modern Physics, Vol. 5 No 4-1, 1-5 (2016). "UDE Cosmology without Higgs boson and without graviton", American Journal of Modern Physics, Vol. 5, No. 4-1, 6-13 (2016), "Energy-mass-gravity theory", American Journal of Modern Physics, Vol. 5, No. 4-1, 20-26 (2016). "Mass-energy equivalence versus Higgs mechanism", American Journal of Modern Physics Vol. 7, No. 4-1, 1-4 (2018). 
[7] Jason Cole, "The imaginary mass Lambda-CDM model", American Journal of Modern Physics Vol. 5, No. 1, 1-14 (2016).

[8] William C. Daywitt, "The trouble with the equations of modern fundamental physics", American Journal of modern physics, Vol. 5 No. 23-32 (2016).

[9] C. J. Isham et al. ed, Quantum Gravity (Oxford University Press, 1975), P. 548, 573.

[10] Adel S. Saada, Elasticity Theory and Applications (Pergamon Press Inc, 1974), P. 331.

[11] V. Fock, The theory of Space Time and Gravitation, Translated from the Russian by N. Kemmer (Pergamon Press, 1959), P. 184-194.,

[12] Claus Kiefer, "Conceptual problem in quantum gravity and quantum cosmology", ISRN Mathematical Physics Vol. 2013, $509316(2013)$

[13] Kazuya Kayama, "Cosmological test of modified gravity", Reports on Progress in Physics Vol. 79, No. 4, 046902 (2016).

[14] S. W. Hawking \& G. F. R. Ellis, The larger Scalar Structure of Space-time (Cambridge University Press. 1973), P. 138.

[15] Bernard Schutz, A First Course in General Relativity, $2^{\text {nd }}$ (Cambridge University Press, 2009), P. 338.

[16] James B. Hartle, Gravity (Pearson Education Asia Limited and Beijing World Publishing Cooperation, 2009), P. 384-395.

[17] Petr Hájíček, An Introduction to the Relativistic Theory of Gravitation (Springer, 2008), P. 160-164, 172.

[18] M. Rashki and S. Jalalzadeh, "Holography from quantum cosmology”, Phys. Rev. D 91, 023501 (2015).

[19] L. N. Granda and D. F. Jimenez, "Dark energy from Gauss-Bonnet and nonminimal couplings", Phys. Rev. D 90, 123512 (2014).

[20] Francisco D. Mazzitelli, et al., "Boundary divergences in vacuum self-energies and quantum field theory in curved space time”, Phys. Rev. D 84, 125008 (2011).

[21] Richard A. Battye and Francesco Pace, "Approximation of the potential in scalar field dark energy models", Phys. Rev. D 94, 063513 (2016)

[22] Oscar Catà, et al., "Dark matter decay through gravity portals", Phys. Rev. D 95, 0350011 (2017).

[23] Michael S. Ref and Christian F. Steinwachs, "Quantum equivalence of $f(R)$ gravity and scalae-tensor theories", Phys. Rev. D97, 044050 (2018).

[24] Hamed Barzegal, et al. "Energy in higher-dimensional spacetime”, Phys. Rev. D 96, 124002 (2017).

[25] Guido D'Amico, et al. “Quantum field theory of interacting dark matter and dark energy: Dark monodromies", Phys. Rev. D 94, 103526 (2016).
[26] Eugeny Babichev, et al. "Bigravitational origin of dark matter", Phys. Rev. D 94, 084055 (2016).

[27] Ding-Yu Chung, "The three postutates of the theory of everyting", Journal of Modern Physics, 2016, 7, 642-655.

[28] Charles V. R. Board and John D. Barrow, "Cosmological models in energy-momentum-squared gravity", Phys. Rev. D 96, 123517 (2017).

[29] Betti Hartmann, et al. "Excited cosmic strings with superconducting currents", Phys. Rev. D 96, 123531 (2017).

[30] Moira I. Gresham, et al. "Early universe synthesis of asymmetric dark matter nuggets", Phys. Rev. D 97, 036003 (2018).

[31] Vincent Desjacques, et al. "Impact of ultralight axion self-interactions on the large scalr structure of the universe", Phys. Rev. D 97, 023529 (2018).

[32] Marco Crisostomi and Kazuya Kayama, "Self-accelerating universe in scalar-tensor theories after GW170817”, Phys. Rev. D 97, 084004 (2018).

[33] Anto. I. Lonappan, et al. "Bayesian evidences for daek energy modes in light of current abservational data", Phys. Rev. D 97, 043524 (2018).

[34] Gaurav Narain and Tianjun Li, "Ultraviolet complete dark energy model”, Phys. Rev. D 97, 083523 (2018).

[35] S. Boran, et al. "GW170817 falsifies dark matter emulators", Phys. Rev. D 97, 041501 (R) (20180).

[36] Cleng-Wei Chiang, et al. "Standard model with a complex scalar singlet: cosmological implications and theoretical considerations", Phys. Rev. D 97, 015005 (2018).

[37] Jonathan Kozaczuk, "Dark photons from nucleal transions". Phys, Rev. D 97, 015014 (2018).

[38] R. Agnese, et al. "Low-mass daek matter search with CDMSlite”, Phys. Rev. D 97, 022002 (2018).

[39] James M. Cline, " $B$ decay anomalies and dark matter from rectorlike confinement”, Phys. Rev. D 97, 015013 (2018).

[40] Steven J. Clank, et al. "Dark matter annihilation into four-body states and implications for the AMS antiproton excess", Phys. Rev. D 97, 023003 (2018).

[41] Vedran Brdar, et al. "Fuzzy dark matter and nonstandard neutrino interactions", Phys. Rev. D 97, 043001 (2018).

[42] Matti Heikinheimo, et al. "prospects for indirect detection of frozen-in dark matter", Phys. Rev. D 97, 063002 (2018).

[43] Weishuang Linda $\mathrm{Xu}$, et al. "Probing sub-Gev dark matter-baryon scattering with cosmological observable" Phys. Rev. D 97, 103530 (2018).

[44] Gérard Gremand, "Universe and matter conjectured as 3-dimensional lattice with topological singularities", Journal of Modern Physics, 2016, 7, 1389-1399. 